\title{
Tissue-specific expression, developmentally and spatially regulated alternative splicing, and protein subcellular localization of OsLpa1 in rice*
}

\author{
Hai-ping $\mathrm{LU}^{1}$, Wei-qin $\mathrm{PANG}^{1}$, Wen-xu $\mathrm{LI}^{1}$, Yuan-yuan TAN ${ }^{1}$, \\ Qing $\mathrm{WANG}^{2}$, Hai-jun $\mathrm{ZHAO}^{1}$, Qing-yao $\mathrm{SHU}^{\dagger \neq 1}$ \\ ( ${ }^{1}$ State Key Laboratory of Rice Biology, Institution of Crop Science, Zhejiang University, Hangzhou 310029, China) \\ $\left({ }^{2}\right.$ Wuxi Hupper Bioseed Technology Academy Ltd., Wuxi 214000, China) \\ ${ }^{\dagger}$ E-mail: qyshu@zju.edu.cn \\ Received Aug. 29, 2015; Revision accepted Nov. 30, 2015; Crosschecked Jan. 13, 2016
}

\begin{abstract}
The OsLpa1 gene (LOC_Os02g57400) was identified to be involved in phytic acid (PA) metabolism because its knockout and missense mutants reduce PA content in rice grain. However, little is known about the molecular characteristics of OsLpa1 in rice and of its homologues in other plants. In the present study, the spatial pattern of OsLpa1 expression was revealed using OsLpa1 promoter::GUS transgenic plants (GUS: $\beta$-glucuronidase); GUS histochemical assay showed that OsLpa1 was strongly expressed in stem, leaf, and root tissues, but in floral organ it is expressed mainly and strongly in filaments. In seeds, GUS staining was concentrated in the aleurone layers; a few blue spots were observed in the outer layers of embryo, but no staining was observed in the endosperm. Three OsLpa1 transcripts (OsLpa1.1, OsLpa1.2, OsLpa1.3) are produced due to alternative splicing; quantitative reversetranscriptase polymerase chain reaction (RT-PCR) analysis revealed that the abundance of OsLpa1.3 was negligible compared with OsLpa1.1 and OsLpa1.2 in all tissues. OsLpa1.2 is predominant in germinating seeds (about 5 times that of OsLpa1.1), but its abundance decreases quickly with the development of seedlings and plants, whereas the abundance of OsLpa1.1 rises and falls, reaching its highest level in 45-d-old plants, with abundance greater than that of OsLpa1.2 in both leaves and roots. In seeds, the abundance of OsLpa1 continuously increases with seed growth, being 27.5 and 15 times greater in 28-DAF (day after flowering) seeds than in 7-DAF seeds for OsLpa1.1 and OsLpa1.2, respectively. Transient expression of chimeric genes with green fluorescence protein (GFP) in rice protoplasts demonstrated that all proteins encoded by the three OsLpa1 transcripts are localized to the chloroplast.
\end{abstract}

Key words: OsLpa1, Low phytic acid, Expression pattern, Alternative splicing, Subcellular localization http://dx.doi.org/10.1631/jzus.B1500205 CLC number: Q946.2

\section{Introduction}

Phytic acid (PA, myo-inositol-1,2,3,4,5,6-hexakisphosphate or $\mathrm{Ins}_{6}$ ) is the primary storage form of phosphorus $(\mathrm{P})$, accounting for $65 \%-85 \%$ of total $\mathrm{P}$ in

\footnotetext{
ॠ Corresponding author

* Project supported by the Sino-German Joint Research Project (No. GZ932), the Key Projects in the National Science \& Technology Pillar Program during the Twelfth Five-year Plan Period (No. 2014BAA 03B04), and the Wuxi Science and Technology Department Funds (No. CLE01N1408), China

(10) ORCID: Qing-yao SHU, http://orcid.org/0000-0002-9201-0593

(C) Zhejiang University and Springer-Verlag Berlin Heidelberg 2016
}

seeds (Raboy, 2003). Under physiological conditions, $\mathrm{InsP}_{6}$ strongly binds to metallic cations forming a mixed salt known as phytate. During seed germination, phytate is broken down to myo-inositol, inorganic phosphate, and minerals for seedling growth. $\mathrm{InsP}_{6}$ can regulate a number of nuclear processes including messenger RNA (mRNA) export, DNA repair, and RNA editing (York, 2006; Monserrate and York, 2010; Lee et al., 2015), and, as a signal molecule, adjust membrane dynamics (di Paolo and de Camilli, 2006; Janetopoulos and Devreotes, 2006; Thole and Nielsen, 2008). However, from a nutritional 
and environmental point of view, $\operatorname{InsP}_{6}$ is a major anti-nutrient for livestock and human nutrition because it cannot be digested by monogastric animals, and it also binds to minerals to reduce their bioavailability (Raboy, 2001). These concerns have together provided a strong impetus for developing low phytic acid (lpa) crops (Stevenson-Paulik et al., 2005; Raboy, 2007). In rice, several lpa mutants have been developed through chemical and physical mutagenesis and the underlying lpa genes (or candidate genes) have been identified, i.e. OsLpal (LOC_Os02g57400) (Kim et al., 2008a; Zhao et al., 2008), OsMRP5 (multidrug resistance protein ATP-binding cassette transporter, LOC_Os03g04920) (Xu et al., 2009), and OsMIK (myo-inositol kinase, LOC_Os03g52760) (Kim et al., 2008b; Zhao et al., 2013).

While other lpa genes have a clear function in either PA biosynthesis or transport, little is known about the biochemical function of OsLpal. Bioinformatics analysis showed that OsLpal has a paralogous gene in rice (LOC_Os09g39870), and one to a few orthologs in other plants, suggesting that OsLpal and its orthologs play active roles in plant biology. However, the only known fact related to its function is that a portion of OsLpal has homology to the P-loop kinase domain of 2-phosphoglycerate kinase (2-PGK) in hyperthermophilic methanogens (Aravind et al., 2000; Kim et al., 2008a; Zhao et al., 2008). The possible roles of OsLpa1 in PA metabolism were discussed by Raboy (2009), but none of them has been experimentally confirmed so far. Hence, more investigations are needed to reveal the molecular characteristics and biological functions of the OsLpal gene and its orthologs in plants.

It is well known that alternative splicing (AS) is a form of post- and co-transcriptional regulation in which pre-mRNA transcripts from the same gene are spliced in different ways, which greatly contributes to the enrichment of eukaryotic transcriptomes (Filichkin et al., 2010; Marquez et al., 2012). For example, $92 \%-94 \%$ of human intron-containing genes and approximately $61 \%$ of Arabidopsis multi-exonic genes can be alternatively spliced (Reddy et al., 2012; Rogers et al., 2012). In the rice transcriptome, AS occurs in $33 \%$ of all rice genes (Zhang et al., 2010). However, little is known about the extent and significance of tissue-specific splicing in rice and other plants (Reddy et al., 2013; Staiger and Brown, 2013).
Seven AS modes of OsLpal were either previously reported (Kim et al., 2008a) or predicted (Zhao et al., 2008) through bioinformatics analysis, or annotated in rice genome databases (Fig. 1a).

Aiming to shed light on OsLpal as well as on AS in plants, in this study we investigated the molecular characteristics of OsLpal in terms of its tissue-specific expression, AS, and protein subcellular localization.

\section{Materials and methods}

\subsection{Generation of transgenic plants and histo- chemical staining}

Analysis of the 5' flanking sequence of OsLpal using the tools PLACE (http://www.dna.affrc.go. jp/PLACE/signalscan.html) and PlantCare (http:// bioinformatics.psb.ugent.be/webtools/plantcare/html) indicated that the promoter region of OsLpal encompasses a $2.1-\mathrm{kb}$ fragment. This fragment was polymerase chain reaction (PCR)-amplified using primer pPro1 (Table 1) in a $25-\mu 1$ volume with $1.0 \mu 1$ DNA $(50 \mathrm{ng} / \mu \mathrm{l}), 0.2 \mathrm{mmol} / \mathrm{L}$ dNTPs, $1.5 \mathrm{mmol} / \mathrm{L}$ $\mathrm{MgSO}_{4}, 0.3 \mu \mathrm{mol} / \mathrm{L}$ each primer, and $0.5 \mu \mathrm{l}$ highfidelity DNA polymerase (TOYOBO, Japan), supplemented with sterile distilled water. The following temperature profile was used: $94^{\circ} \mathrm{C} 2 \mathrm{~min}, 40$ cycles of $94{ }^{\circ} \mathrm{C} 10 \mathrm{~s}, 57^{\circ} \mathrm{C} 30 \mathrm{~s}$, and $68^{\circ} \mathrm{C} 60 \mathrm{~s}$, and a final extension at $68^{\circ} \mathrm{C}$ for $5 \mathrm{~min}$. The resulting amplicons were cloned into the $\mathrm{T}$ vector (TaKaRa, China) after purification. The sequence-verified promoter fragment was then subcloned into the multi-cloning site (MCS) between SalI and BamHI of pCAMBIA1300 and a ProOsLpa1::GUS vector (GUS: $\beta$-glucuronidase) was constructed. The ProOsLpal::GUS vector was introduced into the Agrobacterium tumefaciens strain EHA105 and transferred into the japonica cultivar Xidao No. 1 according to Hiei and Komari (2008).

Transgene positive $T_{0}$ plants were identified by PCR analysis of the hygromycin resistant gene using primer Hyg $^{\mathrm{R}}$ (Table 1) and $\mathrm{T}_{1}$ seeds were harvested and grown into plant lines according to Lu et al. (2015). Root, stem, leaf, flower tissues and seeds (developing and mature) were histochemically stained for $24 \mathrm{~h}$ at $37{ }^{\circ} \mathrm{C}$ with GUS dye solution containing $100 \mathrm{mmol} / \mathrm{L}$ phosphate buffer ( $\mathrm{pH} 7.0), 20 \%(\mathrm{v} / \mathrm{v})$ methanol, $0.5 \%$ $(\mathrm{v} / \mathrm{v})$ Triton X-100, and $0.5 \mathrm{mg} / \mathrm{ml}$ X-Gluc, according to Sieburth and Meyerowitz (1997). 
Table 1 Primers used for molecular cloning and characterization of $O s L p a 1$ in rice

\begin{tabular}{|c|c|c|}
\hline Primer $^{*}$ & Forward $(\mathrm{F})$ and reverse $(\mathrm{R})$ sequences $\left(5^{\prime} \rightarrow 3^{\prime}\right)$ & Amplicon size (bp) \\
\hline \multirow[t]{2}{*}{ pPro1 } & F: GGATCCCAGTAAACTAACCAACGGAGACG & 2100 \\
\hline & R: GTCGACGTCGTCCCCTACCTGCAAAAT & \\
\hline \multirow[t]{2}{*}{$\mathrm{Hgy}^{\mathrm{R}}$} & F: AGAAGAAGATGTTGGCGACCT & 565 \\
\hline & R: GTCCTGCGGGTAAATAGCT & \\
\hline \multirow[t]{2}{*}{$\mathrm{pV} 1$} & F: GCGAAGACCCAAGAGGAA & 453 (gDNA) \\
\hline & R: GACGGCAATGTAGAGGAGC & 366 (cDNA) \\
\hline \multirow[t]{2}{*}{$\mathrm{pV} 2$} & F: ACTCTTAGTAGCATACCCTTCG & 808 (gDNA) \\
\hline & R: CTTCTGTGCCTTCCGTTTTGCC & 424 (cDNA) \\
\hline \multirow[t]{2}{*}{ pRT1 } & F: TCTAGGGAGAAGTTCCTAAGTGTT & 192 \\
\hline & R: ACCCAATCTACTACCCAACAAA & \\
\hline \multirow[t]{2}{*}{ pRT2 } & F: GCACCAGCCATACAGGAAGTGT & 177 \\
\hline & R: CATCAGGAGGATCATCAGCATCT & \\
\hline \multirow[t]{2}{*}{ pRT3 } & F: GCACCAGCCATACAGGAAGTGT & 143 \\
\hline & R: TCATACTGTAAACACACCAATG & \\
\hline \multirow[t]{2}{*}{ pRT-Actin } & F: TGCTATGTACGTCGCCATCCAG & 210 \\
\hline & R: AATGAGTAACCACGCTCCGTC & \\
\hline \multirow[t]{2}{*}{ pSub1.1 } & F: GGCGCGCCCCATGGCGGAGGAGGCGCC & 2058 \\
\hline & R: CCCGGGTGCACACGGCAGTTCTGTGG & \\
\hline \multirow[t]{2}{*}{ pSub1.2 } & F: AGATCT TAATGATGAGAGGCTTTACGGA & 1488 \\
\hline & R: CCCGGGTGCACACGGCAGTTCTGTGG & \\
\hline \multirow[t]{2}{*}{ pSub1.3 } & F: AGATCTTA ATGATGAGAGGCTTTACGGA & 1128 \\
\hline & R: CCCGGGTACTGTAAACACACCAATGCATAC & \\
\hline
\end{tabular}

* Positions of validation PCR (pV1, pV2) and RT-PCR primers (pRT1, pRT2, pRT3) are shown in Fig. 1a

2.2 RNA extraction, reverse transcription, and real-time PCR

To investigate the abundance of the OsLpal transcripts in different tissues during vegetative and reproductive growth, leaf and root tissues of seedlings and plants at different growth stages $(3,14,45$, and $90 \mathrm{~d}$ after germination (DAG)), flowers at heading and seeds of different developmental stages $(7,14,21$, and $28 \mathrm{~d}$ after flowering (DAF)) were collected for total RNA extraction. RNAs were extracted in $1 \mathrm{ml}$ TRIzol reagent according to the manufacturer's instructions (TaKaRa, China). Total RNAs were treated with DNase, and their concentrations and purities were assessed using a Nano Drop 2000 (Thermo Scientific, Wilmington, Delaware, USA). Complementary DNAs (cDNAs) were reverse-transcribed from 500 ng RNA using the M-MLV reverse transcription kit (TaKaRa, China).

To examine whether transcripts other than OsLpa1.1, OsLpa1.2, and OsLpa1.3 exist, two sets of validation primers ( $\mathrm{pV} 1$ and $\mathrm{pV} 2$; Table 1$)$ were designed for PCR of both genomic DNAs (gDNAs) and
cDNAs. Because we were not able to detect transcripts other than those confirmed by Kim et al. (2008a) (see Section Results), subsequent real-time quantitative reverse transcriptase-PCR (qRT-PCR) was carried out only for OsLpa1.1, OsLpa1.2, and OsLpa1.3 using RT primers pRT1, pRT2, and pRT3 (Table 1, Fig. 1a) according to the manufacturer's instructions. Because no part of the OsLpa1.2 transcript is unique compared with OsLpa1.1 and OsLpa1.3, primer pRT2 was designed to amplify the fragment from both OsLpal.1 and OsLpa1.2 (Fig. 1a). The housekeeping gene Actin was used as an internal standard with RT primer pRT-Actin (Table 1). qRT-PCRs were performed in $10 \mu \mathrm{l}$ aliquots, containing $1.0 \mu \mathrm{cDNA}, 0.2 \mu \mathrm{l}$ of each primer $(10 \mu \mathrm{mol} / \mathrm{L})$, and $5.0 \mu 1 \mathrm{mix}$ buffer, supplemented with sterile distilled water, according to the following schedule: $94^{\circ} \mathrm{C} 2 \mathrm{~min}$; 40 cycles of $94{ }^{\circ} \mathrm{C} 15 \mathrm{~s}$ and $60^{\circ} \mathrm{C} 1 \mathrm{~min}$. The relative quantification of gene expression was analyzed using the $2^{-\Delta \Delta C_{\mathrm{T}}}$ method, where $C_{\mathrm{T}}$ value is the cycle number at which the fluorescent signal rises statistically above the background. qRT-PCRs were repeated with three biological replicates. 


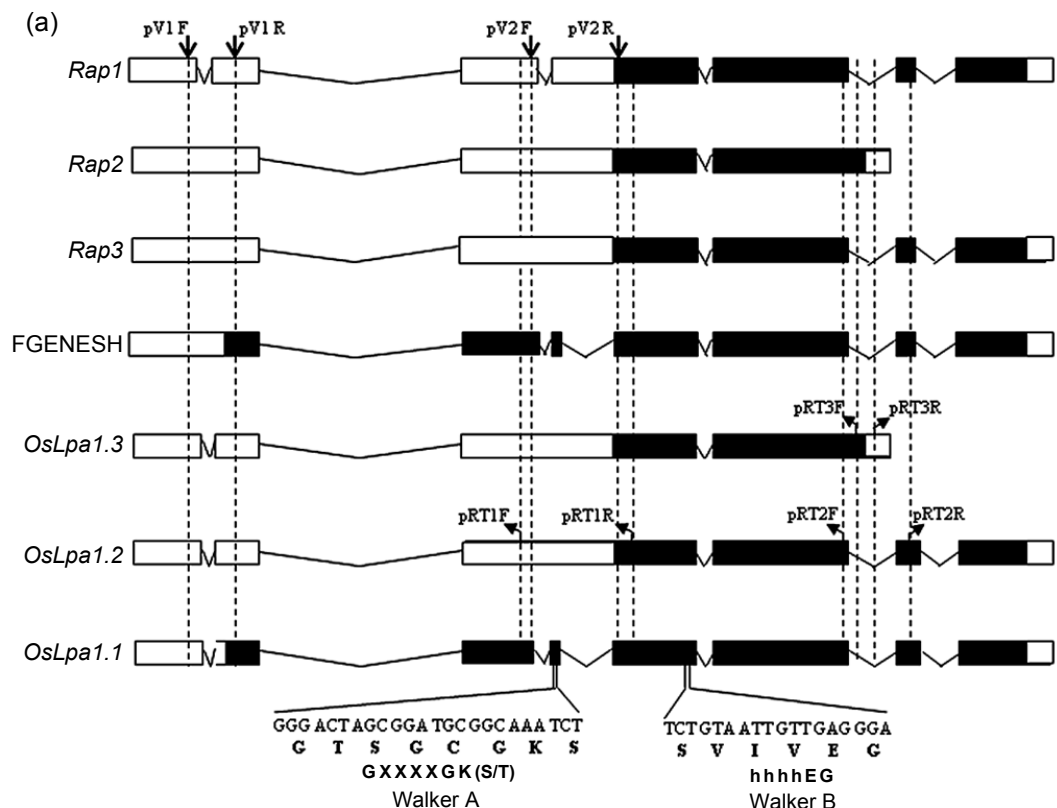

(b)


Fig. 1 Predicted OsLpa1 alternative splicing and translation modes

(a) Three splicing modes (OsLpa1.1, OsLpa1.2, and OsLpa1.3) according to Kim et al. (2008a), one predicted using FGENESH by Zhao et al. (2008), and three transcripts annotated in the RAP database (http://rapdb.dna.affrc.go.jp/viewer/gbrowse/ irgsp1/?name=id:1470481; dbid=annotation:database). Exons (boxes) and introns (lines) are in scales, and empty boxes represent untranslated regions. Primers used for PCRs are shown above with the vertical dotted lines showing their corresponding positions. The characteristic Walker A $(\operatorname{GXXXXGK}(\mathrm{S} / \mathrm{T}))$ and Walker B (hhhhEG) motifs are indicated in the OsLpa1.1; X stands for any amino acid, while $\mathrm{h}$ is a hydrophobic residue. (b) Fragments amplified using validation primers from genomic DNA (gDNA) or reverse-transcribed DNA (complementary DNA (cDNA))

\subsection{Protein subcellular localization}

The full-length coding regions of the three OsLpal transcripts (except for the stop codon) were amplified by PCR using the primers pSub1.1, pSub1.2, and pSub1.3, respectively, from cDNA (Table 1). $\mathrm{PCR}$ products were subcloned into the $\mathrm{T}$-vector, and after sequence verification the target fragment was further cloned into the vector pTZM28-GFP to produce a fusion gene with green fluorescence protein (GFP) under the control of the cauliflower mosaic virus (CaMV) 35S promoter.

For transient expression, Nipponbare seeds were germinated and cultured for $8 \mathrm{~d}$ on $1 / 2 \mathrm{MS}$ culture medium at $28^{\circ} \mathrm{C}$ under continuous lighting. Seedlings were cut into $0.2-\mathrm{cm}$ sections with sterile blades and used for isolation of protoplasts according to Zhang et al. (2011). Ten micrograms of 35S::OsLpa::GFP plasmids were mixed with $220 \mu \mathrm{l} 40 \%(0.4 \mathrm{~g} / \mathrm{ml})$ poly(ethylene glycol) 4000 (PEG-4000) and transformed into $200 \mu \mathrm{l}$ protoplasts by gentle blending. After incubating for $16 \mathrm{~h}$ in the dark, transformed protoplasts were examined under a laser confocal microscope (Leica Microsystems, Bannockburn, IL, USA).

\subsection{Data analysis}

The mean and standard error were calculated from three independent replicates. Statistical analysis was performed using the one-way analysis of variance (ANOVA) program StaView. The differences are considered to be significant when the probability $(P)$ was less than 0.05 .

\section{Results}

\subsection{Expression pattern of OsLpal in rice}

The temporal and spatial patterns of OsLpal expression were determined using transgenic plants that contain the GUS reporter gene driven by the OsLpa1 promoter (OsLpa1::GUS). By PCR analysis of the hygromycin resistant gene using primer $\mathrm{Hyg}^{\mathrm{R}}$ (Table 1), twenty-eight transgene positive plants were identified and were subsequently used for further 
characterization (data not shown). Histochemical staining for GUS activity showed that OsLpal was strongly expressed in stem (Figs. 2a-2c), leaf (Fig. 2d), and root (Fig. 2e). In the floral organ, the filaments were stained dark blue (Figs. $2 \mathrm{f}$ and $2 \mathrm{~g}$ ), but little staining was observed in other parts such as anthers (Fig. 2g) and ovaries (Fig. 2h), indicating OsLpal is expressed mainly in the filaments. In seeds, GUS staining was concentrated in the aleurone layers (Fig. 2i); a few blue spots were observed in the outer layers of embryo (Figs. 2i and 2j), but no staining was observed in the endosperm (Fig. 2i). These observations indicate that OsLpal is mainly expressed in aleurone cells.

\subsection{Developmental and spatial regulation of OsLpa1 alternative splicing}

Among the seven predicted AS modes of OsLpa1, only OsLpa1.1, OsLpa1.2, and OsLpa1.3 were experimentally confirmed by RACE analysis (Kim et al., 2008a). We first performed validation experiments on other possible transcripts using mixed cDNAs from 14 DAG seedlings and developing seeds of 14 DAF. If the Rap2, Rap3, and FGENESH transcripts exist, two fragments would be amplified from cDNAs using the primer $\mathrm{pV} 1$, one with length identical to the fragment from gDNA and one shorter (Fig. 1a). Because we amplified only the expected shorter length amplicons from cDNA (Fig. 1b), we deduce that transcripts of Rap2, Rap 3, and FGENESH do not exist. Similarly by using $\mathrm{pV} 2$ we excluded the existence of Rap1 transcripts because we did not observe fragments with the size predicted for Rap1 transcripts (Fig. 1b).

To reveal whether the AS of OsLpal is regulated spatially and/or developmentally, the relative abundances of its three transcripts were assessed by qRT-PCRs using three sets of transcript-specific primers, i.e. pRT1 for OsLpa1.1, pRT2 for both OsLpa1.1 and OsLpa1.2, and pRT3 for OsLpa1.3 (Fig. 1a). Because very similar amplification efficiencies ( 0.98 vs. 0.99 , respectively) were achieved for primers pRT1 and pRT2, the abundance of OsLpa1.2 can be calculated by deduction of OsLpa1.1 from that measured by using pRT2.

The OsLpa1.3 transcript could be barely detected in leaf and root tissues at all growth stages (Fig. 3a); its abundance was also the lowest in developing seeds, with only about $1 / 4$ that of OsLpa1.2 at its maximum level in seeds of 14 DAF (Fig. 3b).

Both OsLpa1.1 and OsLpa1.2 were abundant compared with OsLpa1.3, their amount being spatially and developmentally regulated (Figs. $3 a$ and $3 b$ ). During the vegetative growth stage, the abundances of OsLpal.1 and OsLpa1.2 changed inversely, i.e., OsLpa1.2 decreased in both leaf and root tissues from germinating seeds (3 DAG), to seedlings (14 DAG), to plants at tillering stage (45 DAG), while OsLpal.1 increased continuously during the same period (Fig. 3a).

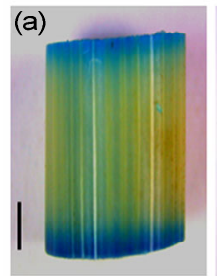

(c)



(d)

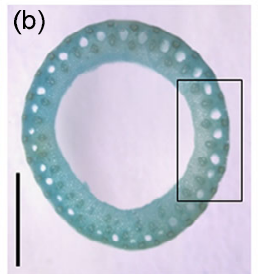

(e)

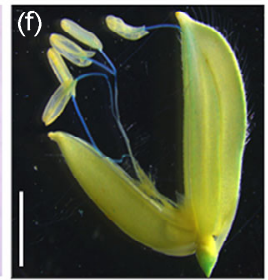

(g)
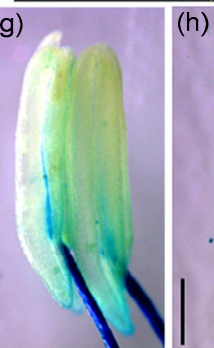



(j)

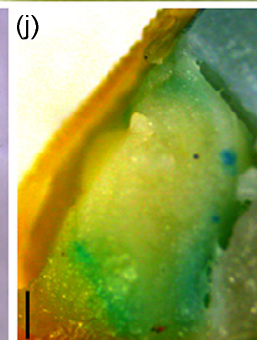

Fig. 2 Histochemical assay of GUS activity in transgenic rice plants expressing the OsLpa1 promoter-GUS fusion gene (a) Stem; (b) Cross-cut of stem; (c) Enlargement of a section of (b) (framed); (d) Leaf segment; (e) Root; (f) A floret; (g) Anther with filaments; (h) Unfertilized ovary with stigmas; (i) Longitudinal cut of a seed; (j) Enlargement of the embryo of (i) (framed). Bars in (a), (b), (d), (e), (f), and (i) equal to $2.0 \mathrm{~mm}$, and those in (c), (g), (h), and (j) $0.5 \mathrm{~mm}$ (Note: for interpretation of the references to color in the text and legend, the reader is referred to the web version of this article) 
Consequently, OsLpa1.2 was 5.7- and 5.2-fold more abundant than OsLpa1.1 in coleoptiles and roots of germinating seeds, respectively, but the amount of OsLpa1.1 became 2.8- and 5.8-fold greater than that of OsLpa1.2 in leaf and root tissues, respectively, at tillering stage. In the flag leaves and roots of plants at flowering stage, the abundances of all three transcripts decreased significantly compared with tillering plants (Fig. 3a). The abundances of both OsLpa1.1 and OsLpa1.2 were also low in unfertilized florets and young seeds but increased significantly in developing seeds starting from 14 DAF. For example, OsLpa1.1 was 11.9- and 27.8-fold more abundant in seeds of 14 and 28 DAF than in those of 7 DAF, respectively. Compared with OsLpa1.1, OsLpa1.2 was less abundant in developing seeds at all stages, although it was also highly abundant (Fig. 3b).
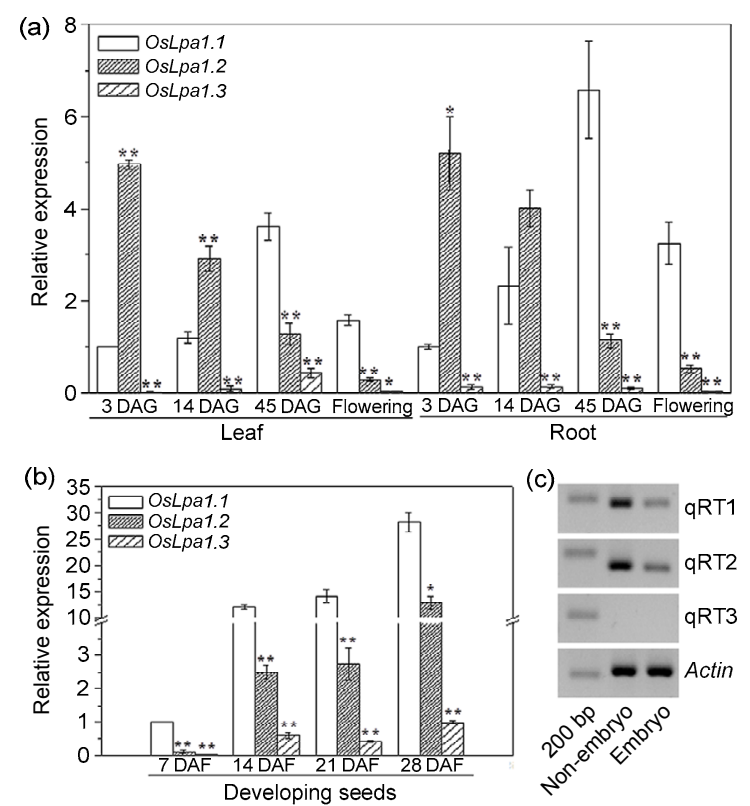

Fig. 3 Abundance analyses of three OsLpa1 transcripts $(\mathrm{a}, \mathrm{b})$ Relative abundances of three OsLpal transcripts in various tissues of plants at different developmental stages, revealed by qRT-PCR analyses. (c) Semi-quantitative RTPCRs of OsLpal in the embryo and non-embryo parts of mature seeds using the three primer sets shown in (a). DAG: days after germination; DAF: days after flowering. Data are mean \pm standard error (SE) of three independent experiments. Relative abundance was calculated using the Actin gene as an internal control and compared with OsLpa1.1 (set as 1) in leaf and root tissues of 3 DAG (a) and seeds of $7 \mathrm{DAF}(\mathrm{b}){ }^{*}$ and ${ }^{* *}$ mean significant differences compared with OsLpal.1 in each stage at $P<0.05$ and $P<0.01$, respectively
Because GUS assay showed that OsLpal is expressed less in the embryo (Fig. 2j), we manually separated embryo from the whole seeds and subjected them to semi-RT-PCR analysis. The result was in accordance with the GUS staining: both OsLpa1.1 and OsLpa1.2 were more profoundly expressed in the non-embryo than in the embryo, and OsLpal.3 was barely detected in either (Fig. 3c).

\subsection{OsLpa1 proteins anchored to the chloroplast}

Through bioinformatic analysis (http://www.cbs. dtu.dk/services/TargetP), a chloroplast localizing signal peptide (45 amino acids) was detected in the protein encoded by OsLpa1.1 at the N terminal, but not in those encoded by OsLpa1.2 and OsLpa1.3. To reveal the localization of proteins encoded by the three OsLpal transcripts, the coding sequences were fused with the GFP gene to generate three chimeric OsLpa1::GFP genes driven by the CaMV $35 \mathrm{~S}$ promoter (Fig. 4a). They were transiently expressed in rice protoplasts for protein localization. Under the confocal microscopy, three chimeric proteins with green fluorescence can completely match the chloroplast with red autofluorescence (Fig. 4b), which indicated that they are all localized to the chloroplast.

\section{Discussion}

Knowledge of tissue-specific expression and protein subcellular localization provides important clues about the function of a gene. Our present study revealed such information about OsLpal in rice, helping to illuminate its role in rice biology and potentially the role of the OsLpal family in other plant species. Recent studies have shown that more than half of these genes in rice undergo AS, either induced by stress conditions or developmentally regulated (E et al., 2013). However, detailed analyses of tissuespecific and developmental AS have so far been very limited (Wang et al., 2014) until our present study which provides new insights into AS of OsLpal in particular and gene AS in general in plants.

Our present study first demonstrated that the AS of OsLpal is regulated both spatially and developmentally through a mechanism yet to be uncovered. 

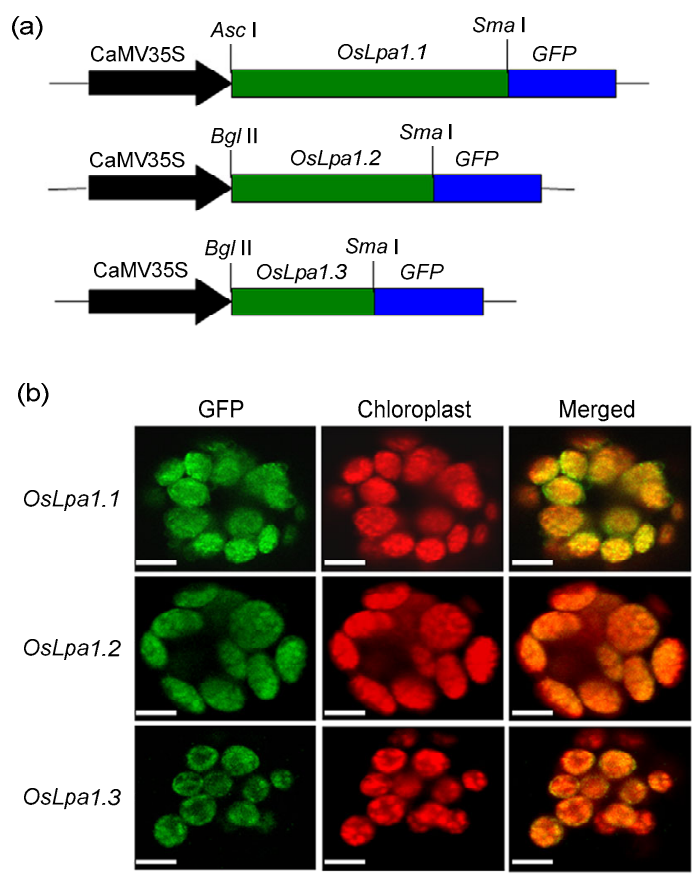

Fig. 4 Subcellular localization of OsLpa1.1, OsLpa1.2, and OsLpa1.3 in rice protoplasts by confocal microscopy (a) The construction of OsLpa1.1, OsLpa1.2, and OsLpa1.3 green fluorescence protein $(G F P)$ fusion genes driven by the CaMV 35S promoter. (b) The green fluorescence from three proteins merged with red fluorescence from the chloroplast using confocal microscopy. Overlay of two images indicated that all three proteins are localized to the chloroplast. The bars equal to $1.5 \mu \mathrm{m}$ (Note: for interpretation of the references to color in this figure legend, the reader is referred to the web version of this article)

The significant and dynamic changes of the abundances of three OsLpal transcripts in different tissues and at different stages suggested that they may play different roles in rice development. The greater abundance of OsLpal.2 compared with OsLpa1.1 in seedling (Fig. 2a), for example, may suggest that the protein encoded by OsLpa1.2 plays a more important role in seedling growth. In contrast, OsLpal.1 may play a more active role than OsLpa1.2 in seed development.

OsLpal was found to be involved in PA metabolism through gene mapping and candidate gene analysis (Kim et al., 2008a; Zhao et al., 2008), with the biochemical function of the protein it encodes being largely unknown. Raboy (2007) discussed three possible roles for OsLpal, i.e. it encodes a 2-phosphoglyceric acid (2-PGA) kinase producing 2,3-PGA2 (which is possibly a competitive inhibitor of the Ins polyphosphate-5-phosphate) or an Ins phosphate kinase (that catalyzes the phosphorylation of Ins monophosphates), or encodes an enzyme that has both of the above functions. However, none of the putative functions has been experimentally confirmed so far. Our present study showed that both OsLpa1.1 and OsLpa1.2 are abundant in developing seeds; it is intriguing to further test whether the two different transcripts encode the two enzymes.

The results of OsLpal expression in different parts of the rice plant provide information about where the OsLpal gene plays a role. In addition to rice seeds, expression of OsLpal was observed in other organs (Fig. 2). In particular, high expression was observed in roots (except the root tip) (Fig. 2e) and in the filaments (Fig. 2g). It is yet to be revealed whether the high expression is related to biosynthesis of PA or other unknown molecules in these tissues. It is well-known that PA is accumulated in both aleurone layers and embryos (Raboy, 2009), and Sato et al. (2011) also showed that LOC_Os02g57400 had the highest expression level in rice Nipponbare embryos (7-42 DAF). However, the promoter::GUS transgenic plant assay showed a high level of OsLpal expression in the aleurone layers and to a lesser extent in the embryo (Figs. 2i and 2j). Therefore our results are not consistent with previous studies and further studies are needed to identify the cause of these differences.

In the present study, we also observed high expression of OsLpal in the filament (Fig. 2g), suggesting that it may play an essential role, possibly in filament elongation at anthesis (Thole et al., 2008; Zhao et al., 2010). This would not be surprising given that two phosphatidylinositol compounds in the lipiddependent PA synthesis pathway, phosphatidylinositol 4,5-bisphosphate $\left(\operatorname{PtdIns}(4,5) \mathrm{P}_{2}\right)$ and phosphatidylinositol 3,4,5-trisphosphate $\left(\operatorname{PtdIns}(3,4,5) \mathrm{P}_{3}\right)$, participate in the recruitment and activation of a wide variety of actin regulatory proteins at the plasma membrane, controlling cell shape, motility, and cytokinesis (di Paolo and de Camilli, 2006).

It is not yet known where PA is synthesized, although it is known that PA biosynthesis starts with the synthesis of $\operatorname{Ins}(3) \mathrm{P}_{1}$. Studies on Arabidopsis indicated that the first steps of PA biosynthesis are carried out in the cytosol and the final steps $\left(\mathrm{InsP}_{3}\right.$ to $\mathrm{Ins}_{6}$ ) in the endoplasmic reticulum (Otegui et al., 
2002). Otegui et al. (2002) also noted that an increase in Mn-phyate in the embryo coincides with higher chlorophyll content. In the present study, we revealed that all proteins are anchored to chloroplast (Fig. 4), suggesting that PA biosynthesis may be related to molecules produced in the chloroplast, mediated or catalyzed by OsLpa1.

Sequence identity searches revealed that OsLpa1.1 has homology to the P-loop kinase domain of 2-PGK. The P-loop NTPase domain super-family is characterized by two conserved nucleotide phosphatebinding motifs, i.e. the Walker A motif (GXXXX $\mathrm{GK}[\mathrm{S} / \mathrm{T}]$, where $\mathrm{X}$ is any residue) and the Walker $\mathrm{B}$ motif (hhhhEG, where $\mathrm{h}$ is a hydrophobic residue). While OsLpa1.1 has both the Walker A and Walker B motifs, OsLpa1.2 and OsLpa1.3 have only the latter (Fig. 1a), suggesting OsLpa1.2 and OsLpa1.3 could be non-functional proteins. Therefore, OsLpa1.2 and OsLpa1.3 or their encoded proteins may function as competitors of OsLpal.1 in transcription as genes involved in stress response (Staiger and Brown, 2013) or in catalysis through formation of homo- and hetero-dimer proteins. In addition, because the $5^{\prime}$ untranslated regions (5' UTRs) could be a part of a promoter (Guo et al., 2010; Suhandono et al., 2014), further studies are needed to reveal the spatiotemporal expression patterns of OsLpal.2 and OsLpa1.3.

\section{Conclusions}

The present study demonstrates that OsLpal was strongly expressed in stem, leaf, and root tissues, and in filaments in the floral organ. In seeds, GUS staining was concentrated in the aleurone layers. Three OsLpa1 transcripts (OsLpa1.1, OsLpa1.2, OsLpa1.3) are produced by AS; quantitative RT-PCR analysis revealed that OsLpa1.2 is predominant in germinating seeds, but its abundance decreases quickly with plant development, whereas the abundance of OsLpa1.1 rises and falls in both leaves and roots. In seeds the abundance of OsLpal continuously increases with seed growth. Transient expression of chimeric genes with GFP in rice protoplasts demonstrated that all proteins encoded by the three OsLpal transcripts are localized to the chloroplast.

\section{Compliance with ethics guidelines}

Hai-ping LU, Wei-qin PANG, Wen-xu LI, Yuan-yuan TAN, Qing WANG, Hai-jun ZHAO, and Qing-yao SHU declare that they have no conflict of interest.

This article does not contain any studies with human or animal subjects performed by any of the authors.

\section{References}

Aravind, L., Wolf, Y.I., Koonin, E.V., 2000. The ATP-CONE: an evolutionarily mobile, ATP-binding regulatory domain. J. Mol. Microbiol. Biotechnol., 2(2):191-194.

di Paolo, G., de Camilli, P., 2006. Phosphoinositides in cell regulation and membrane dynamics. Nature, 443(7112): 651-657. http://dx.doi.org/10.1038/nature05185

E, Z.G., Wang, L., Zhu, J.H., 2013. Splicing and alternative splicing in rice and humans. BMB Rep., 46(9):439-447. http://dx.doi.org/10.5483/BMBRep.2013.46.9.161

Filichkin, S.A., Priest, H.D., Givan, S.A., et al., 2010. Genomewide mapping of alternative splicing in Arabidopsis thaliana. Genome Res., 20(1):45-58. http://dx.doi.org/10.1101/gr.093302.109

Guo, L.L., Yu, Y.H., Xia, X.L., et al., 2010. Identification and functional characterisation of the promoter of the calcium sensor gene $C B L 1$ from the xerophyte Ammopiptanthus mongolicus. BMC Plant Biol., 10(1):18. http://dx.doi.org/10.1186/1471-2229-10-18

Hiei, Y., Komari, T., 2008. Agrobacterium-mediated transformation of rice using immature embryos or calli induced from mature seed. Nat. Protoc., 3(5):824-834. http://dx.doi.org/10.1038/nprot.2008.46

Janetopoulos, C., Devreotes, P., 2006. Phosphoinositide signaling plays a key role in cytokinesis. J. Cell Biol., 174(4):485-490. http://dx.doi.org/10.1083/jcb.200603156

Kim, S.I., Andaya, C.B., Goyal, S.S., et al., 2008a. The rice Oslpal gene encodes a novel protein involved in phytic acid metabolism. Theor. Appl. Genet., 117(5):769-779. http://dx.doi.org/10.1007/s00122-008-0818-z

Kim, S.I., Andaya, C.B., Newman, J.W., et al., 2008b. Isolation and characterization of a low phytic acid rice mutant reveal a mutation in the rice orthologue of maize MIK. Theor. Appl. Genet., 117(8):1291-1301. http://dx.doi.org/10.1007/s00122-008-0863-7

Lee, H.S., Lee, D.H., Cho, H.K., et al., 2015. InsP ${ }_{6}$-sensitive variants of the Gle1 mRNA export factor rescue growth and fertility defects of the ipk1 low-phytic-acid mutation in Arabidopsis. Plant Cell, 27(2):417-431. http://dx.doi.org/10.1105/tpc.114.132134

Lu, H.P., Edwards, M., Wang, Q.Z., et al., 2015. Expression of cytochrome P450 CYP81A6 in rice: tissue specificity, protein subcellular localization, and response to herbicide application. J. Zhejiang Univ.-Sci. B (Biomed. \& Biotechnol.), 16(2):113-122. http://dx.doi.org/10.1631/jzus.B1400168 
Marquez, Y., Brown, J.W.S., Simpson, C., et al., 2012. Transcriptome survey reveals increased complexity of the alternative splicing landscape in Arabidopsis. Genome Res., 22(6):1184-1195. http://dx.doi.org/10.1101/gr.134106.111

Monserrate, J.P., York, J.D., 2010. Inositol phosphate synthesis and the nuclear processes they affect. Curr. Opin. Cell Biol., 22(3):365-373. http://dx.doi.org/10.1016/j.ceb.2010.03.006

Otegui, M.S., Capp, R., Staehelin, L.A., 2002. Developing seeds of Arabidopsis store different minerals in two types of vacuoles and in the endoplasmic reticulum. Plant Cell, 14(6): 1311-1327. http://dx.doi.org/10.1105/tpc. 010486

Raboy, V., 2001. Seeds for a better future: 'low phytate' grains help to overcome malnutrition and reduce pollution. Trend Plant Sci., 6(10):458-462. http://dx.doi.org/10.1016/S1360-1385(01)02104-5

Raboy, V., 2003. myo-Inositol-1,2,3,4,5,6-hexakisphosphate. Phytochemistry, 64(6):1033-1043. http://dx.doi.org/10.1016/S0031-9422(03)00446-1

Raboy, V., 2007. Forward genetics studies of seed phytic acid. Israel J. Plant Sci., 55(2):171-181. http://dx.doi.org/10.1560/IJPS.55.2.171

Raboy, V., 2009. Approaches and challenges to engineering seed phytate and total phosphorus. Plant Sci., 177(4): 281-296. http://dx.doi.org/10.1016/j.plantsci.2009.06.012

Reddy, A.S.N., Roger, M.F., Richardson, D.N., et al., 2012. Deciphering the plant splicing code: experimental and computational approaches for predicting alternative splicing and splicing regulatory elements. Front. Plant Sci., 3:18. http://dx.doi.org/10.3389/fpls.2012.00018

Reddy, A.S.N., Marquez, Y., Kalyna, M., et al., 2013. Complexity of the alternative splicing landscape in plants. Plant Cell, 25(10):3657-3683. http://dx.doi.org/10.1105/tpc.113.117523

Rogers, M.F., Thomas, J., Reddy, A.S.N., et al., 2012. SpliceGrapher: detecting patterns of alternative splicing from RNA-Seq data in the context of gene models and EST data. Genome Biol., 13(1):R4. http://dx.doi.org/10.1186/gb-2012-13-1-r4

Sato, Y., Antonio, B., Namiki, N., et al., 2011. Field transcriptome revealed critical developmental and physiological transitions involved in the expression of growth potential in japonica rice. BMC Plant Biol., 11(1):10. http://dx.doi.org/10.1186/1471-2229-11-10

Sieburth, L.E., Meyerowitz, E.M., 1997. Molecular dissection of the AGAMOUS control region shows that cis elements for spatial regulation are located intragenically. Plant Cell, 9(3):355-365. http://dx.doi.org/10.1105/tpc.9.3.355

Staiger, D., Brown, J.W.S., 2013. Alternative splicing at the intersection of biological timing, development and stress response. Plant Cell, 25(10):3640-3656.

http://dx.doi.org/10.1105/tpc.113.113803

Stevenson-Paulik, J., Bastidas, R.J., Chiou, S.T., et al., 2005. Generation of phytate-free seeds in Arabidopsis through disruption of inositol polyphosphate kinases. PNAS, 102(35):12612-12617.

http://dx.doi.org/10.1073/pnas.0504172102

Suhandono, S., Apriyanto, A., Ihsani, N., 2014. Isolation and characterization of three cassava elongation factor $1 \alpha$ (MeEF1A) promoters. PLoS ONE, 9(1):e84692. http://dx.doi.org/10.1371/journal.pone.0084692

Thole, J.M., Nielsen, E., 2008. Phosphoinositides in plants: novel functions in membrane trafficking. Curr. Opin. Plant Biol., 11(6):620-631. http://dx.doi.org/10.1016/j.pbi.2008.10.010

Thole, J.M., Vermeer, J.E.M., Zhang, Y.L., et al., 2008. ROOT HAIR DEFECTIVE4 encodes a phosphatidylionositol-4phosphate phosphatase required for proper root hair development in Arabidopsis thaliana. Plant Cell, 20(2): 381-395. http://dx.doi.org/10.1105/tpc.107.054304

Wang, L., Cao, C.L., Ma, Q.B., et al., 2014. RNA-seq analyses of multiple meristems of soybean: novel and alternative transcripts, evolutionary and functional implications. BMC Plant Biol., 14:169. http://dx.doi.org/10.1186/1471-2229-14-169

Xu, X.H., Zhao, H.J., Liu, Q.L., et al., 2009. Mutations of the multi-drug resistance-associated protein $\mathrm{ABC}$ transporter gene 5 result in reduction of phytic acid in rice seeds. Theor. Appl. Genet., 119(1):75-83. http://dx.doi.org/10.1007/s00122-009-1018-1

York, J.D., 2006. Regulation of nuclear processes by inositol polyphosphates. Biochim. Biophys. Acta, 1761(5-6):552-559. http://dx.doi.org/10.1016/j.bbalip.2006.04.014

Zhang, G.J., Guo, G.W., Hu, X.D., et al., 2010. Deep RNA sequencing at single base-pair resolution reveals high complexity of the rice transcriptome. Genome Res., 20(5): 646-654.

http://dx.doi.org/10.1101/gr.100677.109

Zhang, Y., Su, J.B., Duan, S., et al., 2011. A high efficient rice green tissue protoplast system for transient gene expression and studying light/chloroplast-related process. Plant Methods, 7(1):30. http://dx.doi.org/10.1186/1746-4811-7-30

Zhao, H.J., Liu, Q.L., Ren, X.L., et al., 2008. Gene identification and allele-specific marker development for two allelic low phytic acid mutations in rice (Oryza sativa L.). Mol. Breeding, 22(4):603-612. http://dx.doi.org/10.1007/s11032-008-9202-6

Zhao, H.J., Cui, H.R., Xu, X.H., et al., 2013. Characterization of $O s M I K$ in a rice mutant with reduced phytate content reveals an insertion of a rearranged retrotransposon. Theor. Appl. Genet., 126(12):3009-3020. http://dx.doi.org/10.1007/s00122-013-2189-3

Zhao, Y., Yan, A., Feijo, J., et al., 2010. Phosphoinositides regulate clathrin-dependent endocytosis at the tip of 
pollen tubes in Arabidopsis and tobacco. Plant Cell, 22(12):4031-4044.

http://dx.doi.org/10.1105/tpc.110.076760

\section{中文概要}

题 目: 水稻低植酸基因 OsLpal 可变剪接和表达的时空 特征及编码蛋白的亚细胞定位

目 的: 揭示水稻低植酸基因 OsLpal 的分子生物学特征, 特别是深化对其可变剪切和表达的时空和组织 特征, 以及蛋白亚细胞定位的认识。

创新点: 确定了 OsLpal 存在的三种剪切方式, 明确了三 种转录本在不同组织和发育时期丰度的变化; 揭 示了 OsLpal 表达的组织和时空差异, 确定其在 根、种子糊粉层细胞和花丝中高度表达; 明确了 三种转录本编码的蛋白均定位于亚叶绿体。
方 法: 通过培育 OsLpal 启动子与 $\beta$-葡萄糖醛酸糖苷酶 (GUS) 杂合基因的转基因植株, 通过不同组织 的 GUS 组织化学染色确定 OsLpal 表达的组织特 异性; 通过设计特异性引物确定 OsLpal 存在的 转录方式, 采用实时荧光定量聚合酶链式反应 (PCR) 分析三种转录本在不同组织和发育时期 的丰度; 采用 OsLpal 三种转录本与绿色荧光蛋 白（GFP）基因构建杂合基因并在水稻原生质体 中的瞬时表达, 在共聚焦显微镜下观察蛋白的亚 细胞定位。

结 论: OsLpal 在根、茎、叶和花丝有强烈的表达。它存 在三种可变剪切方式, 产生的三种转录本存在明 显的时空和组织差异, 但其编码的蛋白均定位于 叶绿体。

关键词: OsLpa1; 低植酸; 表达模式; 可变剪接; 亚细胞 定位 